\title{
Fluorescence Depolarization Study on Local Motions of Anthracene-Labeled Poly(alkyl methacrylate)s in Dilute Solutions and Evaluation of Their Chain Stiffness
}

\author{
Takashi SaSaKi, Haruyuki Arisawa, and Masahide Yamamoto* \\ Department of Polymer Chemistry, Kyoto University, Kyoto 606, Japan
}

(Received September 3, 1990)

\begin{abstract}
Samples of Poly(methyl methacrylate) (PMMA), poly(ethyl methacrylate) (PEMA), and poly(isopropyl methacrylate) (PiPMA) labeled with anthracene in the middle of the backbone chain were synthesized, and their local motions in dilute solutions were investigated by the fluorescence depolarization technique. Both the static stiffness parameter $s^{-1}$ and the mean relaxation time $T_{\mathrm{m}}$ as a measure of the dynamic chain stiffness were evaluated from the time-resolved data of fluorescence anisotropy ratio, and a correlation between these two parameters was found. Conformational energy calculations were performed for the diad compounds of PMMA, PEMA, and PiPMA, and then, the characteristic ratios were calculated according to the rotational isomeric state (RIS) model. The static chain stiffness parameter $\lambda^{-1}$ was obtained for the above three polymers as an independent evaluation of the static chain stiffness by a comparison between the RIS model and the helical wormlike chain model established by Yamakawa and his co-workers, and a correlation between $\lambda^{-1}$ and $T_{\mathrm{m}}$ was also found. The conformational character of PiPMA is remarkably different from that of PMMA and PEMA, resulting in greater chain stiffness for PiPMA than for PMMA and PEMA.

KEY WORDS Fluorescence Depolarization / Anthracene-Labeled Poly(methyl methacrylate) / Anthracene-Labeled Poly(ethyl methacrylate) / Anthracene-Labeled Poly(isopropyl methacrylate) / Local Motions / Conformational Energy Calculation / RIS Model / Chain Stiffness /
\end{abstract}

Recently, local chain dynamics of flexible polymers has been studied in detail by the fluorescence depolarization technique as well as by other experimental tools. An advantage of the fluorescence depolarization method lies in the direct observation of the time-dependent orientation auto-correlation function (second order) of a finite bond vector, when the sample polymer is labeled with anthracene group in the middle of the backbone chain. This method provides a critical comparison between theory and experiment, and therefore, diagnostic information for the theory can be obtained. Viovy et al. ${ }^{1}$ tested various theoretical models for the local chain dynamics by the fluorescence depolarization measurements on anthracenelabeled polystyrene in dilute solutions. Recen- tly, we investigated the local motions of anthracene-labeled poly(methyl methacrylate) (PMMA) in some solvents. ${ }^{2,3}$ Our previous result ${ }^{3}$ showed that the local conformational motions belonging to the low-friction limit of Kramers can be well explained by the discrete models in which the discrete motional modes are taken into account.

On the other hand, various theoretical approaches have been developed to understand the local conformational relaxations of polymer chains. These approaches may be divided into two types. The first type is to simulate a real polymer chain by a fairly simplified (idealized) model with some characteristic parameters which can be determined experimentally (phenomenological parameters). The 
individual characters of various polymers are reflected on those phenomenological parameters, and one can evaluate chain stiffness from these parameters. The second one is to construct a model that contains information about detailed conformational characters of individual polymers. Such a theoretical approach usually meets some mathematical difficulties, so that crude approximations must be employed. However, this type of approach is becoming of importance as the interests in polymer chain dynamics have been concentrated to how the motions of macromolecules reflect their chemical structures. Though it seems complicated to describe the Brownian motions of real polymer chains in terms of their exact chemical structures, the subject is fascinating, and especially, it is remarkable that local conformational motions are considered to be significantly affected by the individual ehain characters (local chemical structures) compared with large-scale motions.

From an experimental point of view, it is important to investigate local motions of various polymers by the fluorescence depolarization method with precise analysis for the elucidation of the relation between the local motions and the chemical structures. The fluorescence emission anisotropy data may be analyzed by the theories of the first type in order to obtain the phenomenological model parameters and the mean relaxation time for various systems. In this case, the obtained parameters reflect the individual chain characters, so one can evaluate the chain stiffness.

Nevertheless, the fluorescence depolarization studies on the local main chain dynamics by the time-resolving method have been done for limited kinds of polymers until now, i.e., polystyrene, ${ }^{1}$ PMMA, ${ }^{2,3}$ polybutadiene, ${ }^{4}$ and polyisoprene. ${ }^{5}$ Anufrieva and her co-workers ${ }^{6}$ investigated the effect of the ester alkyl group of various poly(alkyl methacrylate)s on the local relaxation time, and found that the local relaxation time is enhanced with the increase of the length of the ester group. However, their experimental data were obtained by a conventional method of continuous excitation, thus, an exact evaluation of the mean relaxation time or the chain stiffness was not given.

In the present paper, we studied precisely the effect of the ester group on the local chain mobility and the chain stiffness of three poly(alkyl methacrylate)s, i.e., PMMA, poly(ethyl methacrylate) (PEMA), and poly(isopropyl methacrylate) (PiPMA) by the nanosecond time-resolving technique. The samples used in this study are well defined ones, which are labeled with anthracene group in the middle of the backbone chain as

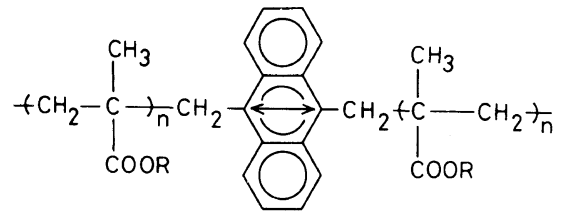

where $\mathrm{R}$ denotes methyl, ethyl, or isopropyl group. The transition moment indicated by the arrow lies parallel to the backbone of the polymer chain. In this case, we can observe directly the auto-correlation function of the main chain bond at the middle position by the fluorescence depolarization measurements. ${ }^{7}$

We first discuss the dynamic chain stiffness of the three polymers, i.e., PMMA, PEMA, and PiPMA, are discussed in terms of the mean relaxation time obtained from the timeresolving measurements of fluorescence depolarization. The static chain stiffness parameter $s^{-1}$, which is evaluated from the phenomenological parameters of the Hall-Helfand $(\mathrm{HH})$ model, ${ }^{8}$ is also discussed. Next, the result of conformational energy calculations are presented to reveal the conformational characters of the three polymers. From the results of the energy calculations, we evaluate the static chain stiffness parameter $\lambda^{-1}$, as another estimation of the static chain stiffness by a comparison between the rotational isomeric state (RIS) model $^{9}$ and the helical wormlike (HW) chain model established by Yamakawa and his co-workers. ${ }^{10}$ Further, we discuss the relation- 
ship between the static and dynamic chain stiffness parameters.

\section{EXPERIMENTAL}

Anthracene-labeled PMMA was prepared in the same manner as described previously. ${ }^{3}$ Anthracene-labeled PEMA and anthracenelabeled PiPMA were also prepared in a manner similar to that for the PMMA. Anionic polymerization of ethyl methacrylate (EMA) or isopropyl methacrylate (iPMA) was initiated with (1,1-diphenyl 3-methylpentyl)lithium in $\mathrm{THF}$ at $-78^{\circ} \mathrm{C}$. After the exhaustion of the monomer was completed, the living ends were deactivated by a small amount of the bifunctional terminator, 9,10-bis(bromomethyl)anthracene at $-78^{\circ} \mathrm{C}$. The anionic polymerization of EMA and iPMA were affected significantly by the impurities contained in the monomers, so that they had to be purified carefully by use of triethylaluminium. ${ }^{11}$ The obtained polymers were precipitated in hexane several times, and then, they were dried in vacuo. For PEMA and PiPMA, further purification by GPC fractionation had to be done to eliminate the end-labeled fraction.

The absorption and emission spectra of the anthracene labels revealed no photophysical interactions between the attached chains and the chromophore. Also, the fluorescence intensities decayed with single exponential functions for all the samples. The obtained fluorescence lifetimes are listed in Table I. Molecular weights and triad tacticities (racemo fraction $f_{r}$ ) of the obtained polymers were determined by GPC and ${ }^{13} \mathrm{C}$ NMR, respectively, as listed in Table II. As shown in this table, the obtained polymers are predominantly syndiotactic.

The sample polymers were dissolved in ethyl acetate and methylcellosolve (2-methoxyethanol). The concentration of all these solutions was kept to be $0.1 \mathrm{wt} \%$, and at this concentration, the chromophore (anthracene group) concentration was less than $10^{-5} \mathrm{~mol}$ -
Table I. Fluorescence lifetime of the samples (ns)

\begin{tabular}{lcc}
\hline \multirow{2}{*}{ Sample } & \multicolumn{2}{c}{ Solvent } \\
\cline { 2 - 3 } & $\begin{array}{c}\text { Ethyl acetate } \\
\left(25^{\circ} \mathrm{C}\right)\end{array}$ & $\begin{array}{c}\text { Methylcellosolve } \\
\left(26^{\circ} \mathrm{C}\right)\end{array}$ \\
\hline PMMA-1 & 10.7 & 10.9 \\
PEMA-1 & 10.6 & 10.8 \\
PiPMA & 10.8 & 10.7 \\
\hline
\end{tabular}

Table II. Molecular weights and racemi fractions of the samples

\begin{tabular}{lccc}
\hline \multicolumn{1}{c}{ Sample } & $M_{n} \times 10^{-4}$ & $M_{w} \times 10^{-4}$ & \multicolumn{1}{c}{$f_{r}$} \\
\hline PMMA-1 & 2.1 & 2.3 & 0.93 \\
PEMA-1 & 2.1 & 2.5 & 0.89 \\
PiPMA & 2.5 & 2.8 & 0.87 \\
PMMA-2 & 12.8 & 15.0 & 0.93 \\
PEMA-2 & 15.7 & 17.7 & 0.89 \\
\hline
\end{tabular}

$1^{-1}$. These solutions were degassed before measurements were taken.

Time-resolving measurements of fluorescence depolarization were done by the nanosecond single-photon counting system in our laboratory. ${ }^{3}$ The obtained data were analyzed by the method of nonlinear leastsquares fitting as described in the previous paper. $^{3}$

\section{RESULTS AND DISCUSSION}

We first present the result of the averaged anisotropy ratio $\bar{r}$ as a rough estimation of the chain mobility, though it also contains information of the fluorescence intensity decay. The averaged anisotropy ratio can be obtained from the data of the time-resolving measurement as ${ }^{12}$

$$
\bar{r}=\frac{\int_{0}^{\infty}\left[I_{\|}(t)-G I_{\perp}(t)\right] \mathrm{d} t}{\int_{0}^{\infty}\left[I_{\|}(t)+2 G I_{\perp}(t)\right] \mathrm{d} t}
$$

where $I_{\|}(t)$ and $I_{\perp}(t)$ are the parallel and 
Table III. Averaged anisotropy ratio $\bar{r}$

\begin{tabular}{lcc}
\hline \multirow{2}{*}{ Sample } & \multicolumn{2}{c}{ Solvent } \\
\cline { 2 - 3 } & $\begin{array}{c}\text { Ethyl acetate } \\
\left(25^{\circ} \mathrm{C}\right)\end{array}$ & $\begin{array}{c}\text { Methylcellosolve } \\
\left(26^{\circ} \mathrm{C}\right)\end{array}$ \\
\hline PMMA-1 & $0.048 \pm 0.004$ & $0.128 \pm 0.005$ \\
PEMA-1 & $0.042 \pm 0.004$ & $0.120 \pm 0.006$ \\
PiPMA & $0.055 \pm 0.004$ & $0.137 \pm 0.009$ \\
PMMA-2 & $0.062 \pm 0.002$ & $0.150 \pm 0.006$ \\
PEMA-2 & $0.053 \pm 0.003$ & $0.147 \pm 0.006$ \\
\hline
\end{tabular}

perpendicular components of the fluorescence emission intensity, respectively, and $G$ is the compensating factor for the anisotropic sensitivity of the photon detector. We obtained the averaged anisotropy ratio by summing up the single-photon counting data according to eq 1 . As shown in Table III, the averaged anisotropy ratio obtained here slightly depends on the molecular weight. The data for the lower molecular weight samples (PMMA-1, PEMA-1, and PiPMA) show that $\bar{r}(\mathrm{PiPMA}) \geq \bar{r}(\mathrm{PMMA})>$ $\bar{r}$ (PEMA). One can also notice that $\bar{r}$ (PMMA) $>$ $\bar{r}$ (PEMA) for the higher molecular weight samples.

Now, we estimated two kinds of chain stiffness (dynamic and static ones) from our data. The dynamic chain stiffness represents how long the memory of the initial orientation of a segment persists (time). On the other hand, the static chain stiffness represents how far the orientational correlation extends along the chain (length).

An exact estimation of the dynamic chain stiffness (local chain mobility) can be done in terms of the mean relaxation time $T_{\mathrm{m}}$ defined as $^{7}$

$$
T_{\mathrm{m}}=r_{0}^{-1} \int_{0}^{\infty} r(t) \mathrm{d} t
$$

where $r(t)$ is the time-dependent fluorescence anisotropy ratio defined by

$$
r(t)=\frac{I_{\|}(t)-G I_{\perp}(t)}{I_{\|}(t)+2 G I_{\perp}(t)}
$$

and $r_{0}$ is the initial anisotropy ratio, i.e., $r_{0}=$ $r(0)$. It is unable to estimate $T_{\mathrm{m}}$ by the conventional method of the continuous excitation measurement. To estimate the value of $T_{\mathrm{m}}$, we first found an experimental function made up of the sum of exponential decay terms for each observed anisotropy decay by the leastsquares fitting method. Then, the integration of this function in eq 2 was calculated. The results are listed in Table IV. Again, we see that $T_{\mathrm{m}}($ PiPMA $)>T_{\mathrm{m}}($ PMMA $)>T_{\mathrm{m}}($ PEMA $)$, namely, the dynamic chain stiffness is PiPMA > PMMA > PEMA. Anufrieva et al. ${ }^{6}$ showed that the relaxation time increases slightly with the length of the ester group in polar media, and this may be inconsistent with the present result. However, their result does not contain the data for PEMA and PiPMA.

The mean relaxation time $T_{\mathrm{m}}$ also contains information about the solvent quality. Some experimental results ${ }^{13-15}$ showed that the local mobility in dilute solution is larger in good solvents than in the theta solvent. Waldow et al. ${ }^{15}$ attributed this phenomenon to a higher local segment density in poor solvents than in good solvents. In general, poly(alkyl methacrylate) becomes more soluble in the solvents used in this study as the ester group becomes larger. Therefore, the fact that $T_{\mathrm{m}}$ (PMMA) $>$ $T_{\mathrm{m}}$ (PEMA) may be partially due to such solubility difference. In either case, the difference in bulkiness of the ester group between PMMA and PEMA does not seem to have a significant effect on the dynamic chain stiffness.

Next, we estimated the static chain stiffness from the anisotropy decay data. Our previous study $^{2,3}$ on the local chain dynamics of PMMA showed that the discrete models such as the $\mathrm{HH}$ model, the generalized diffusion and loss (GDL) model (improved HH model), ${ }^{1}$ and the Jones-Stockmayer (JS) model ${ }^{16}$ well explain the local conformational transition processes. The expression for the auto-correlation functions of these models involve the modified Bessel function (the JS model provides the 
Table IV. Stiffness parameters obtained from the time-resolved data of fluorescence depolarization

\begin{tabular}{|c|c|c|c|c|}
\hline \multirow{2}{*}{ Sample } & \multicolumn{2}{|c|}{$\begin{array}{l}\text { Ethyl acetate } \\
\qquad\left(25^{\circ} \mathrm{C}\right)\end{array}$} & \multicolumn{2}{|c|}{$\begin{array}{l}\text { Methylcellosolve } \\
\qquad\left(26^{\circ} \mathrm{C}\right)\end{array}$} \\
\hline & $T_{\mathrm{m}} / \mathrm{ns}$ & $s^{-1}$ & $T_{\mathrm{m}} / \mathrm{ns}$ & $s^{-1}$ \\
\hline PMMA-1 & $2.62 \pm 0.31$ & $1.11 \pm 0.11$ & $10.1 \pm 0.6$ & $1.72 \pm 0.22$ \\
\hline PEMA-1 & $2.09 \pm 0.44$ & $1.11 \pm 0.16$ & $8.3 \pm 0.8$ & $1.48 \pm 0.27$ \\
\hline PiPMA & $2.84 \pm 0.44$ & $1.25 \pm 0.16$ & $11.8 \pm 1.1$ & $1.74 \pm 0.38$ \\
\hline PMMA-2 & $3.61 \pm 0.22$ & $1.32 \pm 0.07$ & $15.6 \pm 0.8$ & $2.20 \pm 0.27$ \\
\hline PEMA-2 & $2.79 \pm 0.25$ & $1.34 \pm 0.09$ & $15.1 \pm 0.8$ & $1.96 \pm 0.27$ \\
\hline
\end{tabular}

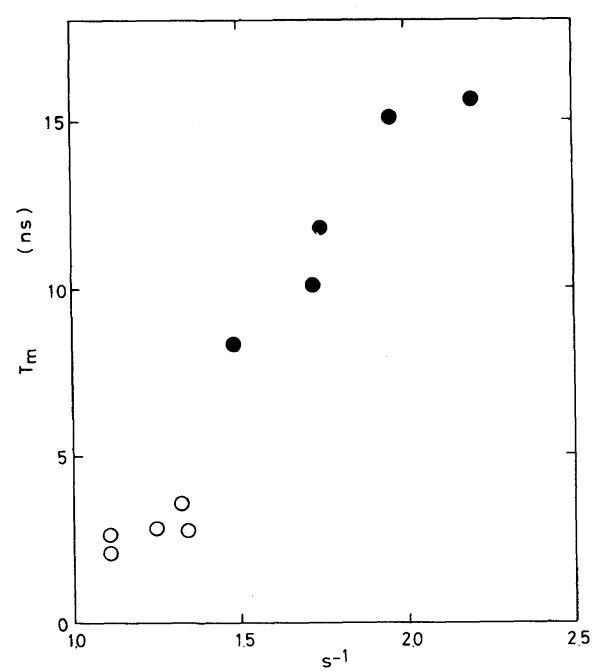

Figure 1. Plot of the mean relaxation time $T_{\mathrm{m}}$ against the static stiffness parameter $s^{-1}$. (O), methylcellosolve; (O), ethyl acetate.

modified Bessel function within a certain limit ${ }^{17}$ ). We proposed the parameter $s^{-1}$ as a measure of the static stiffness, ${ }^{3}$ and this is expressed in terms of the dynamic (phenomenological) parameters of the $\mathrm{HH}$ model as

$$
s^{-1}=1 /\left[\cosh ^{-1}\left(1+T_{2} / T_{1}\right)\right]
$$

where $T_{1}$ and $T_{2}$ are the correlation times corresponding to the independent (isolated) and correlated transitions, respectively. The parameter $s^{-1}$ represents the extent of motional correlation (coupling range), namely, $s^{-1}$ is a measure of the static chain stiffness scaled by the number of motional units. This is essentially a phenomenological parameter, and therefore, should depend on the solvent viscosity, polymer compatibility with solvents, and temperature. The obtained values of $s^{-1}$ are also listed in Table IV. We plotted $T_{\mathrm{m}}$ against $s^{-1}$ in Figure 1. As shown in this figure, there is an apparent correlation between the static and dynamic chain stiffness. Recently, Yamakawa et $a l .{ }^{18}$ showed the correlation between the static and dynamic chain stiffness, and they presented an explanation for the result.

The fact that both the static and dynamic chain stiffness parameters of PMMA are larger than those of PEMA suggests that the difference in the ester group between these polymers should not be simply attributed to the difference in their steric bulkiness. Hence, consideration on detailed chemical structure is necessary to explain the present result. For this purpose, as will be shown in the next section, we first performed the conformational energy calculations for the diad model compounds of the corresponding polymers. Based on these results, we calculated the characteristic ratios by the method of the RIS model, and then, we estimated the parameter $\lambda^{-1}$ as another static chain stiffness parameter from an independent source, using the theory of the HW chain model. We employed such a calculation method to estimate the value of $\lambda^{-1}$ for convenience, because the experimental determination is not so easy. The other model parameters of the HW chain were also determined simultaneously. 


\section{CONFORMATIONAL ENERGY CALCULATIONS AND ESTIMATION OF THE STATIC CHAIN STIFFNESS}

\section{Conformational Energy Calculations}

We calculated the intramolecular energies of the diad model compounds of MMA (methyl methacrylate), EMA, and iPMA. The calculated energies consist of the contributions from non-bonded interactions between atoms, bond angle deformations, inherent torsional potentials, and the Coulombic interactions between partially charged atoms contained in the ester groups. As for PMMA, the conformational energy calculation was already performed by Sundararajan and Flory ${ }^{19}$ (the paper is referred to as SF henceforth). The present calculations were done according to their manner as will be described below. All the calculation procedures were performed on digital computers FACOM M-382 and M-780 at the Data Processing Center in Kyoto University.

Non-bonded interactions between atoms were calculated by the Lennard-Jones potential function $E_{i j}=a_{i j} r^{-12}-b_{i j} r^{-6}$, where the constant $a_{i j}$ was determined in such a way that $E_{i j}$ takes the minimum value when the interatom distance $r$ is just the sum of the van der Waals radii for atoms $i$ and $j$. The constant $b_{i j}$ was estimated by the Slater-Kirkwood formula. $^{20}$

The energy due to the deformation of the bond (valence angle) was calculated by the formula $E(\theta)=A(\Delta \theta)^{2}$, where $\Delta \theta$ is the distortion of the bond angle $\theta$, and $A$ is a force constant. The inherent torsional potential energy for the backbone bond was calculated according to the three-fold potential with barrier height of $2.8 \mathrm{kcal} \mathrm{mol}^{-1} .{ }^{9}$ The Coulombic interaction was estimated for the bond dipole moments of $\mathrm{C}-\mathrm{O}$ and $\mathrm{C}=\mathrm{O}$.

The geometrical parameters for the diads of MMA used in the calculations were the same as those employed in the SF. The geometrical parameters of the most stable conformations
Table V. Geometrical parameters of the ester groups found by energy calculations

\begin{tabular}{lll}
\hline & \multicolumn{1}{c}{ Bond angle $^{\mathrm{a}}$} & $\mathrm{deg}$ \\
\hline Ethyl group & $\angle \mathrm{OCC}$ & 110.0 \\
Isopropyl group & $\angle \mathrm{C}^{\mathrm{b}} \mathrm{O}^{\mathrm{d}} \mathrm{C}^{\mathrm{e}}$ & 117.3 \\
& $\angle \mathrm{O}^{\mathrm{d}} \mathrm{C}^{\mathrm{e}} \mathrm{C}^{\mathrm{f}, \mathrm{g}}$ & 108.6 \\
& $\angle\left(\mathrm{C}^{\mathrm{b}} \mathrm{O}^{\mathrm{d}} \mathrm{C}^{\mathrm{e}}\right)\left(\mathrm{O}^{\mathrm{d}} \mathrm{C}^{\mathrm{e}} \mathrm{C}^{\mathrm{f}, \mathrm{g}}\right)$ & $104.0,133.0$ \\
\hline
\end{tabular}

${ }^{\text {a }}$ Superscripts affixed to the atoms are specified in Figure 2.

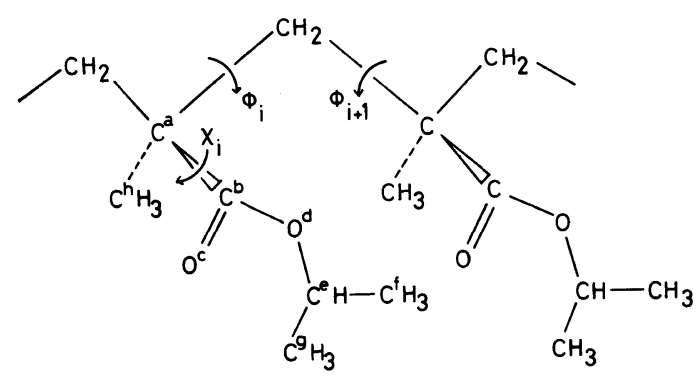

Figure 2. Geometrical structure of the isotactic poly(isopropyl methacrylate) chain in the trans-trans ( $t t)$ state.

Table VI. Conformations and energies at their minimum points for the diad model compounds

\begin{tabular}{cccc}
\hline \multicolumn{1}{c}{ Diad } & $\phi_{i} / \mathrm{deg}$ & $\phi_{i+1} / \mathrm{deg}$ & $E_{\mathrm{min}} / \mathrm{kcal} \mathrm{mol}^{-1}$ \\
\hline MMA meso & $20(-15)$ & $-15(20)$ & 19.4 \\
racemo & 10 & 10 & 19.3 \\
EMA meso & $20(-15)$ & $-15(20)$ & 18.2 \\
racemo & 10 & 10 & 18.1 \\
iPMA meso & 105 & 10 & 16.5 \\
& 10 & 105 & 16.6 \\
racemo & 5 & 5 & 14.5 \\
\hline
\end{tabular}

for the ethyl and isopropyl groups were obtained by energy calculations as listed in Table V. For MMA, the carbon and oxygen atoms of $\mathrm{C}^{\mathrm{h}} \mathrm{C}^{\mathrm{a}} \mathrm{C}^{\mathrm{b}} \mathrm{O}^{\mathrm{c}} \mathrm{O}^{\mathrm{d}} \mathrm{C}^{\mathrm{e}}$ lie in the same plane $\left(\chi_{i}=0^{\circ}\right.$ in Figure 2), and the ester carbonyl bond lies in the eclipsed position with the $\alpha$-methyl group. The latter is consistent with the result of the SF that such eclipsed conformation for the ester carbonyl is more stable than the opposite conformation $\left(\chi_{i}=180^{\circ}\right)$. We have confirmed from energy calculations that this 
tendency was also the case for EMA and iPMA.

Table VI shows the locations and energies at the minimum points. The rotation angles $\phi_{i}$ and $\phi_{i+1}$ are taken according to the manner specified in ref $21\left[\phi_{i}=\phi_{i+1}=0^{\circ}\right.$ for the trans-trans ( $t t)$ state]. As for the meso diad of iPMA, the lowest energy minimum appears at $\left(\phi_{i}, \phi_{i+1}\right)=\left(105^{\circ}, 10^{\circ}\right)$ [the gauche-trans $(g t)$ conformation], and merely shallow hollows appear in the region of the $t t$ conformation. This remarkable feature is due to the strong repulsion between the isopropyl groups at the $t t$ conformation. As shown in Table VI, the lowest energy for the racemo diad of iPMA is lower than that for the meso diad.

\section{Statistical Weights}

The results of the conformational energy calculations clearly showed that the conformational energies in the gauche $(-)(\bar{g})$ region are fairly high compared with the $t$ and $g$ conformations, therefore, the $\bar{g}$ conformation can be omitted as pointed out in the SF.

However, recent studies on the conformational energy calculations ${ }^{22,23}$ which reanalyzed the conformation character of PMMA considering the torsional rotation of the ester group (varying the angle $\chi_{i}$ ), showed that the $\bar{g}$ conformation is stabilized when the ester group rotates to a certain proper position. The energy parameters obtained by such analyses were also scrutinized in comparison with the experimental data, ${ }^{23}$ and the statistical weights of the RIS model were calculated according to the three- or six-state model including the $\bar{g}$ state, then the characteristic ratios were estimated. ${ }^{22,23}$ These analyses concluded that it is more desirable to consider the $\bar{g}$ con- formation than to use the two-state model of the SF. In this study, however, we employ the simple two-state model, which seems to be sufficient for the purpose to evaluate the static chain stiffness parameter $\lambda^{-1}$. For more detailed discussion on the conformational character of poly(alkyl methacrylate)s, the precise model containing $\bar{g}$ state must be employed, and this is now under investigation.

The elements of the statistical weight matrix of the RIS model were calculated in the same manner as that of the SF for PMMA and PEMA. Partition function $z_{\mathrm{s}}$ (subscript $\mathrm{s}$ denotes $t t, m, g t, m$, etc.) for each conformational state of the diad was obtained by taking the sums of Boltzmann factors of the energies at intervals of $5^{\circ}$ over the respective domain. ${ }^{19}$ Here, we chose the temperature to be $300 \mathrm{~K}$. The obtained values are listed in Table VII. For PMMA and PEMA, the partition functions are divided by $z_{t t, m}$. The normalized statistical weight matrixes for the two-state model become 19,21 $^{19}$

$$
\begin{aligned}
& U^{\prime}=\left(\begin{array}{ll}
1 & 1 \\
1 & 0
\end{array}\right) \\
& U_{m}^{\prime \prime}=\left(\begin{array}{ll}
1 & \alpha \\
\alpha & \alpha^{2} / \beta
\end{array}\right) \\
& U_{r}^{\prime \prime}=\left(\begin{array}{ll}
\beta & \alpha \\
\alpha & \alpha^{2} / \beta
\end{array}\right)
\end{aligned}
$$

where

$$
\begin{gathered}
\alpha=z_{g t, m} / z_{t t, m} \\
\beta=z_{t t, r} / z_{t t, m}
\end{gathered}
$$

The values of the partition functions for PMMA obtained here, are slightly different

Table VII. Partition function for each conformational state at $300 \mathrm{~K}$

\begin{tabular}{lrrrrrrr}
\hline & $z_{t t, \boldsymbol{m}}$ & $z_{g t, \boldsymbol{m}}$ & $z_{t g, \boldsymbol{m}}$ & $z_{g g, \boldsymbol{m}}$ & $z_{t t, \boldsymbol{r}}$ & $z_{g t, \boldsymbol{r}}$ & $z_{g g, \boldsymbol{r}}$ \\
\hline PMMA & 10.59 & 0.56 & 0.56 & 0.02 & 15.93 & 0.55 & 0.02 \\
PEMA & 9.65 & 0.53 & 0.53 & 0.01 & 16.28 & 0.62 & 0.02 \\
PiPMA & 0.16 & 10.12 & 11.99 & 0.38 & 472.9 & 20.16 & 0.37 \\
\hline
\end{tabular}


from those of the SF, but this disagreement may be due to trivial errors which caused in the practical procedures on the computers, and this does not make any significant problem.

The temperature dependence of $\alpha$ and $\beta$ can be assumed as ${ }^{19,21}$

$$
\begin{aligned}
& \alpha=\alpha_{0} \exp \left(-E_{\alpha} / R T\right) \\
& \beta=\beta_{0} \exp \left(-E_{\beta} / R T\right)
\end{aligned}
$$

where $E_{\alpha}=\left\langle E_{g t, m}\right\rangle-\left\langle E_{t t, m}\right\rangle$, and $E_{\beta}=\left\langle E_{t t, r}\right\rangle-$ $\left\langle E_{t t, m}\right\rangle .\left\langle E_{\mathrm{s}}\right\rangle$ can be calculated according to the equation ${ }^{19}$

$$
\left\langle E_{\mathrm{s}}\right\rangle=z_{\mathrm{s}}^{-1} \sum_{\phi_{i}, \phi_{i+1}} E_{\phi_{i}, \phi_{i+1}} \exp \left(-E_{\phi_{i}, \phi_{i+1}} / R T\right)
$$

Using eq $8-12$, we obtained $\alpha_{0}$ and $\beta_{0}$. The summation in eq 12 for the calculation of $\left\langle E_{\mathrm{s}}\right\rangle$ was done at an interval of $5^{\circ}$ both for $\phi_{i}$ and $\phi_{i+1}$. These results were used to calculate the characteristic ratios as described below.

\section{Characteristic Ratios}

Characteristic ratio $C_{n}$ defined as $C_{n}=\left\langle R^{2}\right\rangle_{0} /$ $n l^{2}$, was calculated by the usual method ${ }^{9}$ with the statistical weight matrixes obtained above. Here, $\left\langle R^{2}\right\rangle_{0}$ denotes the mean-square end-toend distance, and $n$ and $l$ are the number of bonds and the bond length, respectively. The calculations of $C_{n}$ were performed by use of the FORTRAN program based on the RIS model written by Yamakawa and his coworkers.

For PMMA and PEMA, as was done in the $\mathrm{SF}$, we adjusted the values of $E_{\alpha}$ and $E_{\beta}$ so as to coincide the limiting values of the characteristic ratio $C_{\infty}$, with those obtained by experiments (intrinsic viscosity measurements ${ }^{24-26}$ ). In this case, $\alpha_{0}$ and $\beta_{0}$ were fixed to the values obtained above. The result of the adjustment is shown in Table VIII.

For PiPMA, these are no experimental determinations of $C_{\infty}$ both for the isotactic and syndiotactic ones so far. So, we employed the values of $\alpha$ and $\beta$ obtained directly from eq 8 and 9 (with no adjustment); $\alpha=70$, and $\beta=3.0 \times 10^{3}$ at $300 \mathrm{~K}$.

In this way, we calculated $C_{n}$ for $n=1$ to 1000 at $300 \mathrm{~K}$ for the three polymers of isotactic and syndiotactic ones. Next, we generated the Monte Carlo chains of heterotactic for the three polymers, and calculated $C_{n}$ in the same way. This Monte Carlo procedure is also contained in the FORTRAN program mentioned above. In this calculation, the values of racemo fraction $f_{r}$ were fixed to those of our samples, i.e., 0.93 for PMMA, 0.89 for PEMA, and 0.87 for PiPMA.

\section{Determination of the Static Chain Stiffness Parameter}

The model parameters of the HW chain are the curvature constant and the torsion constant of the characteristic helix $\kappa_{0}$ and $\tau_{0}$, respectively, the static chain stiffness $\lambda^{-1}$, the Poisson ratio $\sigma$, and the shift factor $M_{L}$ (the molecular weight per unit contour length). These parameters can be determined by a comparison with some physical quantities such as the characteristic ratio and the angular correlation functions calculated from the RIS model. The parameters for various kinds of polymers have been determined by this method. ${ }^{27}$ Recently,

Table VIII. Energy parameters used in the RIS calculations

\begin{tabular}{lcccccc}
\hline & & & & \multicolumn{2}{c}{ at $300 \mathrm{~K}$} \\
& $\alpha_{0}$ & $E_{\alpha} / \mathrm{cal} \mathrm{mol}^{-1}$ & $\beta_{0}$ & $E_{\beta} / \mathrm{cal} \mathrm{mol}^{-1}$ & $\alpha$ & $\beta$ \\
\hline PMMA & 1.6 & 1150 & 1.4 & -600 & 0.232 & 3.83 \\
PEMA & 2.4 & 1090 & 1.9 & -600 & 0.389 & 5.20 \\
PiPMA & & & & & 70 & 3000 \\
\hline
\end{tabular}


an attempt of experimental determination has been done, ${ }^{28}$ and the obtained parameters are considered to be more reliable than those estimated from the comparison between the RIS and the HW theories. Indeed, the calculated characteristic ratios based on the RIS model do not always agree with the experimental data, so, we adjusted the energy parameters. As a result, our obtained characteristic ratios for PMMA and PEMA contain some phenomenological information. This means that the physical quantities obtained directly from the RIS model with no adjustment have some uncertainty. The origin of this uncertainty may be brought by a somewhat crude approximation of the twostate model. Indeed, recent conformational energy calculations of PMMA considering the torsional rotation of the ester carbonyl give consistent result with experiments for the characteristic ratio. ${ }^{22}$

In any way, the most desirable way to determine those model parameters seems to be the one depending only on experimental data such as the anisotropic light scattering. ${ }^{29}$ However, such experiments must be carefully performed, and also the sample preparation is not so easy. Thus, we have made up our minds to employ the calculation method as a convenient way.

The RIS calculation with the statistical weight matrixes gives not only $C_{n}$ but also the angular correlation function. ${ }^{30}$ One can also estimate the model parameters from the analysis of this quantity. In this study, however, we analyzed only the characteristic ratio merely for the sake of convenience. We plotted $C_{n} / C_{\infty}$ from the RIS calculation against $\log x(x=n / 2)$, and also plotted the theoretical values of the HW chain model varying the model parameters to achieve the best fitting.

Table IX shows the obtained best-fit model parameters at $300 \mathrm{~K}$. The parameters of PMMA have been already obtained by the calculation method, ${ }^{10,27}$ and our present values do not show a significant inconsistency with the previous results. The values of $\lambda^{-1}$ show that the static chain stiffness becomes larger in the order PMMA, PEMA, PiPMA for the isotactic chains $\left(f_{r}=0\right)$, PEMA, PMMA, PiPMA for the syndiotactic chains $\left(f_{r}=1\right)$. Also, it is worth noticing that the syndiotactic chain is stiffer than the isotactic one for all the three polymers.

The values of partition function listed in Table VII qualitatively indicate that the racemo diads of both MMA and EMA are more likely to take the $t t$ conformation in the equilibrium state than their meso diads, leading to the larger static chain stiffness for the syndiotactic chains than the isotactic ones. In addition, the tendency to take the $t t$ conformation leads to the helical nature for the syndiotactic chains. The values of $\kappa_{0}$ and $\tau_{0}$ show that the syndiotactic PMMA and syndiotactic PEMA belong to the domain I defined in ref 27, which corresponds to a region for the typical HW chain.

Table IX. Best-fit parameters of the HW chain model and characteristic ratio $C_{\infty}$ at $300 \mathrm{~K}$

\begin{tabular}{clccccc}
\hline Sample & $f_{t}$ & $\kappa_{0}$ & $\tau_{0}$ & $\lambda^{-1} / \AA$ & $M_{L} / \AA^{-1}$ & $C_{\infty}$ \\
\hline PMMA & 0 & 1.3 & 1.0 & 23.4 & 38.1 & 10.0 \\
PEMA & 1 & 4.0 & 1.0 & 58.5 & 38.3 & 7.65 \\
& 0 & 1.6 & 1.1 & 27.8 & 46.0 & 9.88 \\
PiPMA & 1 & 3.6 & 0.9 & 52.5 & 43.3 & 8.00 \\
PMMA & 0 & 0.8 & 2.0 & 71.8 & 56.4 & 32.2 \\
PEMA & 1 & 11 & 1.0 & 135 & 49.7 & 3.24 \\
PiPMA & 0.93 & 2.1 & 0.7 & 47.2 & 37.7 & 8.52 \\
& 0.89 & 3.3 & 1.3 & 42.2 & 42.8 & 9.33 \\
\end{tabular}


As for the racemo diad, we can see also qualitatively from the partition functions that the tendency of taking the $t t$ conformation is slightly stronger for MMA than for EMA, i.e., $z_{t t, r} / z_{g t, r}$ (MMA) $>z_{t t, r} / z_{g t, r}($ EMA). The relatively high probability of taking the $t t$ conformation for the racemo diad of MMA is due to the instability of the $g t$ state compared to the $t t$ state. However, the reason for this is not clear at present. This leads to the result that the static chain stiffness of the syndiotactic PMMA is larger than that of the syndiotactic PEMA.

The static chain stiffness of PiPMA is fairly larger both for the isotactic and syndiotactic ones compared with that of PMMA and PEMA. This may reflect a considerably high steric effect of the isopropyl group which brings about an expansion of energy difference between each conformational state. The meso diad of iPMA is most stable at the $g t$ conformation, and of course, the values of partition function indicate stronger tendency of taking the $t t$ conformation for the racemo diad than the meso diad, leading to $\lambda^{-1}$ (isotactic) $<\lambda^{-1}$ (syndiotactic). However, even the isotactic chain of PiPMA is relatively stiffer than PMMA and PEMA of the isotactic ones in spite of its relatively low probability of taking the $t t$ conformation. This suggests that the long sequence of even the $t g$ (or $g t$ ) conformation also increases the static chain stiffness.

Figures 3, 4, and 5 show plots of the reduced characteristic ratio $C_{n} / C_{\infty}$ against $\log x$ for the PMMA, PEMA, and PiPMA, respectively, of the racemo fractions adjusted to those of the present samples. Table IX also shows the best-fit model parameters for these three polymers. We can see that there is a fairly good agreement between the RIS model and the HW chain model. The curve for PMMA shows an apparent peak near $x=15$ which is peculiar to the syndiotactic chain. ${ }^{31}$ This is due to a cyclic conformation produced by a sequence of the $t t$ conformation. This tendency seems to be reduced for PEMA and PiPMA (for PiPMA,

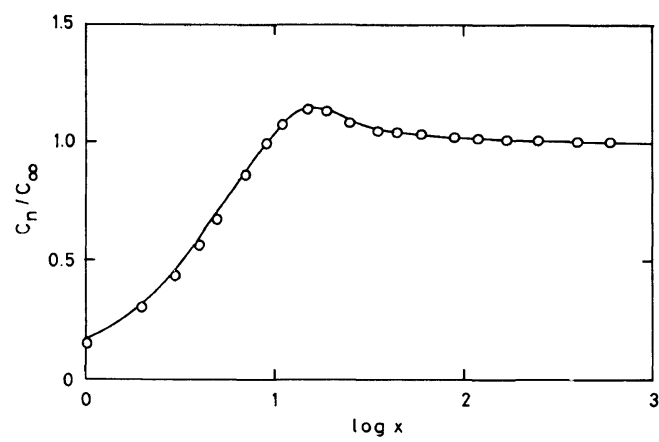

Figure 3. Reduced characteristic ratio $C_{n} / C_{\infty}$ plotted against $\log x$ for the Monte Carlo PMMA chain $\left(f_{r}=0.93\right)$. Values obtained from the RIS model are plotted with circles. Solid curve indicates the theoretical values from the HW chain model.

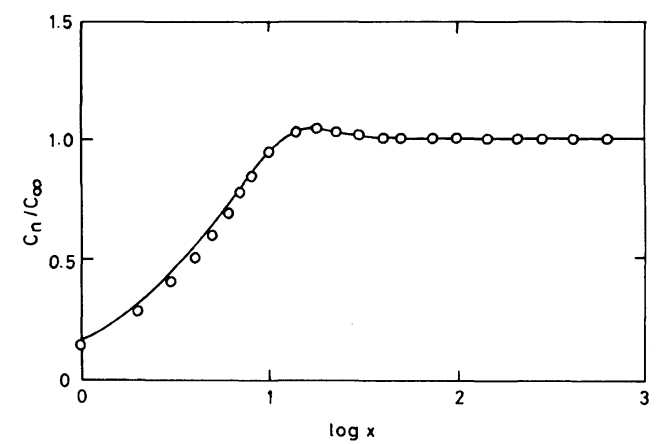

Figure 4. Reduced characteristic ratio plotted against $\log x$ for the Monte Carlo PEMA chain $\left(f_{r}=0.89\right)$.

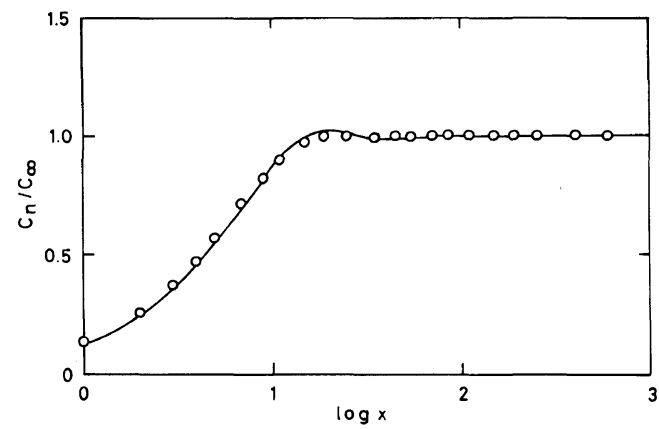

Figure 5. Reduced characteristic ratio plotted against $\log x$ for the Monte Carlo PiPMA chain $\left(f_{r}=0.87\right)$.

merely a small peak is discernible). However, it can be said that these three polymers of the adjusted racemo fractions essentially have the 
helical nature. The values of $\kappa_{0}$ and $\tau_{0}$ for these polymers indicate that these three polymers belong to the domain $I$ defined in ref 27 .

\section{FURTHER DISCUSSION}

The values of $\lambda^{-1}$ listed in Table IX show that the static chain stiffness is PiPMA $>$ PMMA > PEMA for the polymers of the adjusted racemo fractions. This result is roughly consistent to our experimental result for $s^{-1}$, and also means that there is a correlation between $\lambda^{-1}$ and $T_{\mathrm{m}}$. Figure 6 shows a relationship between these parameters.

Thus, we made sure of a correlation between the static and dynamic chain stiffness for our poly(alkyl methacrylate)s from another source, i.e., $\lambda^{-1}$, though the correlation is rather weak. This correlation for poly(alkyl methacrylate)s may be understood by considering two factors, i.e., bulkiness effect of the ester group, and the tacticity effect. Bulkiness of the ester group

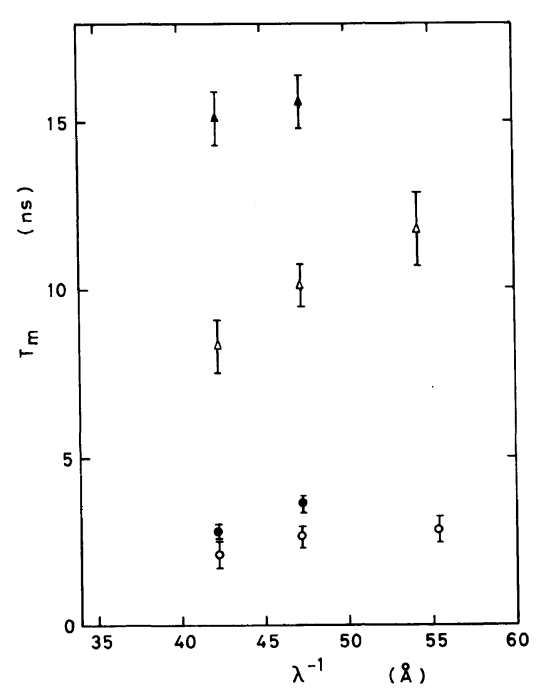

Figure 6. Plot of the mean relaxation time $T_{\mathrm{m}}$ against the static chain stiffiness parameter $\lambda^{-1} .(\triangle)$, PMMA-1, PEMA-1, and PiPMA (low molecular weight) in methylcellosolve; (O), PMMA-1, PEMA-1, and PiPMA (low molecular weight) in ethyl acetate; $(\boldsymbol{\Delta})$, PMMA-2 and PEMA-2 (high molecular weight) in methylcellosolve; (O), PMMA-2 and PEMA-2 (high molecular weight) in ethyl acetate. enhances the asymmetrical feature of the geometry of the segment of poly(alkyl methacrylate)s, and this may lead to an expansion of the energy difference between the stable conformational states. As a result, the population of the specified (most stable) conformation increases in the chain, and therefore, the static chain stiffness increases. On the other hand, the bulkiness of the ester group also increases the steric hindrance for the conformational transition, i.e., the energy barrier for the transition becomes higher, and therefore, $T_{\mathrm{m}}$ becomes larger. Thus, the bulkiness of the ester group increases both the static and dynamic chain stiffness.

Tacticity (racemo fraction) of the polymer has a significant effect upon the chain stiffness. If the difference in the bulkiness is not so effective as we have already seen for the case of PMMA and PEMA, the tacticity dependence on the chain stiffness plays a significant role. From the result of $\lambda^{-1}$ (Table IX), we can see that the relative chain stiffness of PMMA and PEMA is strongly dependent on the tacticity. We should care about the configurational character of the polymer chain when we estimate the chain stiffness.

At this stage, we discuss the relationship between the two static chain stiffness parameters, $s^{-1}$ and $\lambda^{-1}$. Of course, we have determined $s^{-1}$ experimentally, so our obtained values of $s^{-1}$ involve phenomenological information. On the other hand, the values of $\lambda^{-1}$ estimated above are semi-empirical as mentioned before. However, a remarkable point of difference is that $s^{-1}$ is derived from the $\mathrm{HH}$ model which assumes that the local motions of polymer chain consist of two idealized modes of transition, i.e., the isolated and correlated transitions. These two transitions are based on an implicit assumption that the smallest motional unit of a certain size exists. The dimension of $s^{-1}$ is the number of motional units (dimensionless), and $s^{-1}$ means the number of motional units along the chain to which the motional correlation extends from the observed 
segment. On the contrary, the parameter $\lambda^{-1}$, which is in the dimension of (absolute) length, means directly a correlation length along the chain, and can be derived with no assumption of the motional unit. Thus, comparing the two static chain stiffness parameters, we need to take into account the above difference. Information on the size of the motional unit assumed in the $\mathrm{HH}$ model is necessary to convert $s^{-1}$ to the quantity of length.

In the present study, the mean relaxation time $T_{\mathrm{m}}$ obtained from the time-resolving fluorescence depolarization measurements shows that $T_{\mathrm{m}}(\mathrm{PiPMA})>T_{\mathrm{m}}(\mathrm{PMMA})>T_{\mathrm{m}}(\mathrm{PEMA})$, the racemo fractions of which are $0.87,0.93$, and 0.89 , respectively. We plotted $T_{\mathrm{m}}$ against $s^{-1}$ which represents a static chain stiffness on the basis of the $\mathrm{HH}$ model, and a correlation between these two parameters was observed.

We also obtained the values of $\lambda^{-1}$ as another static chain stiffness parameter from a different source, i.e., a comparison of the RIS model with the HW chain model. Plot of $T_{\mathrm{m}}$ against $\lambda^{-1}$ also shows a correlation, and this fact convinces us that a correlation between the static and dynamic chain stiffness exists for the treated polymers in this study.

Furthermore, it is revealed that the static chain stiffness depends fairly strongly on the tacticity of the polymer chain. The stiffness difference between PMMA and PEMA, the conformational characters of which resemble each other, may be subject to the racemo fraction. This means that characterization of the chain configuration is inevitable for the discussion of chain stiffness. For PiPMA, the bulkiness of the ester group has a remarkable effect especially on the conformational character of the meso diad (high energy for the $t t$ conformation). This feature is clearly different from that of PMMA and PEMA. As a result, the stiffness parameter of PiPMA is larger (both static and dynamic) than the other two polymers.

The observed correlation between the static and dynamic chain stiffness for the present polymers may reflect not only the bulkiness effect, but also the tacticity effect. It has already been pointed out ${ }^{18}$ that in general, the correlation between the static and dynamic chain stiffness can be drawn if the activation energy for the conformational transition is almost independent of the kind of polymer chains. At present, there seems to be little unambiguous experimental evidence for this assumption, and therefore, further experimental investigations on various polymer chains with different experimental tools are required to solve this problem.

Acknowledgments. We thank Prof. $\mathrm{H}$. Yamakawa for kind permission to use the FORTRAN program written in his laboratory, in the calculations of the RIS model, and we also thank his collaborators for valuable suggestions through the estimation of $\lambda^{-1}$. Prof. T. Higashimura and Dr. M. Sawamoto are acknowledged for characterizing our samples by GPC. This work was supported in part by a Grant-in-Aid for Scientific Research (No. 62470094) from the Ministry of Education, Science, and Culture of Japan.

\section{REFERENCES}

1. J. L. Viovy, L. Monnerie, and J. C. Brochon, Macromolecules, 16, 1845 (1983).

2. T. Sasaki, M. Yamamoto, and Y. Nishijima, Makromol. Chem., Rapid Commun., 7, 345 (1986).

3. T. Sasaki, M. Yamamoto, and Y. Nishijima, Macromolecules, 21, 610 (1988).

4. J. L. Viovy, L. Monnerie, and F. Melora, Macromolecules, 18, 1130 (1985).

5. V. Veissier, J. L. Viovy, and L. Monnerie, Polymer, 30, 1262 (1989).

6. E. V. Anufrieva and Yu. Ya. Gotlib, Adv. Polym. Sci., 40, 1 (1981), and papers cited therein.

7. B. Valeur and L. Monnerie, J. Polym. Sci., Polym. Phys. Ed., 14, 11 (1976).

8. C. K. Hall and E. Helfand, J. Chem. Phys., 77, 3275 (1982).

9. P. J. Flory, "Statistical Mechanics of Chain Molecules," Interscience Publishers, New York, 1969.

10. H. Yamakawa and M. Fujii, J. Chem. Phys., 64, 5222 (1976), and succeeding papers. 
11. R. D. Allen, T. E. Long, and J. E. McGrath, Polym. Bull., 15, 127 (1986).

12. A. Jablonski, Bull. Acad. Pol. Sci., Ser. Sci. Math. Astr. Phys., 8, 259 (1960).

13. D. Phillips, "Polymer Photophysics-Luminescence, Energy Migration and Molecular Motion in Synthetic Polymers," Chapman \& Hall, London, New York, 1985, Chapter 6.

14. T. Sasaki and M. Yamamoto, unpublished result.

15. D. A. Waldow, B. S. Johnson, P. D. Hyde, M. D. Ediger, T. Kitano, and K. Ito, Macromolecules, 22, 1345 (1989).

16. A. A. Jones and W. H. Stockmayer, J. Polym. Sci., Polym. Phys. Ed., 15, 847 (1977).

17. Y. Y. Lin, A. A. Jones, and W. H. Stockmayer, $J$. Polym. Sci., Polym. Phys. Ed., 22, 2195 (1984).

18. H. Yamakawa, T. Yoshizaki, and M. Fujii, J. Chem. Phys., 84, 4693 (1986).

19. P. R. Sundararajan and P. J. Flory, J. Am. Chem. Soc., 96, 5025 (1974).

20. J. C. Slater and J. G. Kirkwood, Phys. Rev., 37, 682 (1931).
21. P. J. Flory, P. R. Sundararajan, L. C. DeBolt, J. Am. Chem. Soc., 96, 5015 (1974).

22. M. Vacatello and P. J. Flory, Macromolecules, 19, 405 (1986).

23. P. R. Sundararajan, Macromolecules, 19, 415 (1986).

24. G. V. Schulz and R. Z. Kirste, Phys. Chem. (Frankfurt am Main), 30, 171 (1961).

25. R. Jenkins and R. S. Porter, Polymer, 23, 105 (1982).

26. S. N. Chinai and R. J. Valles, J. Polym. Sci., 39, 363 (1959).

27. M. Fujii, K. Nagasaka, J. Shimada, and $H$. Yamakawa, Macromolecules, 16, 1613 (1983).

28. T. Konishi, T. Yoshizaki, J. Shimada, and H. Yamakawa, Macromolecules, 22, 1921 (1989).

29. H. Yamakawa, M. Fujii, and J. Shimada, J. Chem. Phys., 71, 1611 (1979).

30. H. Yamakawa and J. Shimada, J. Chem. Phys., 70, 609 (1979).

31. D. Y. Yoon and P. J. Flory, Polymer, 16, 645 (1975). 